\title{
THE INFLUENCE OF GAS-PHASE HYDROGENATION PARAMETERS ON THE PROCESSES OF ACTIVATION, SORPTION AND ACCUMULATION OF HYDROGEN IN THE POWDER OF PURE TITANIUM
}

\author{
$V$. N. Kudiiarov ${ }^{1,}{ }^{*}, M$. N. Babihina ${ }^{1}$, and D.V. Gvozdyakov ${ }^{1}$ \\ ${ }^{1}$ National Research Tomsk Polytechnic University, 634050 Tomsk, Russia
}

\begin{abstract}
The technique for hydrogenation of powder hydrogen-storage materials based on gas-phase hydrogenation method has been developed in this work. The using of developed technique allow prepare pure titanium powder samples with hydrogen concentration in range from 0.5 to 4 wt.\%. It was showed, that pressure increasing from 0.5 to 2 atm at constant temperature $400{ }^{\circ} \mathrm{C}$ leads to decreasing of powder activation time from 140 to $15 \mathrm{~s}$. Temperature increasing on $50{ }^{\circ} \mathrm{C}$ and more degrees leads to significant decreasing of powder activation time. Pressure increasing from 0.5 to 2 atm leads to increasing of hydrogen sorption rate and concentration in 1.27 and 1.19 times respectively. Temperature increasing from 400 to $550{ }^{\circ} \mathrm{C}$ leads to increasing of hydrogen sorption rate in 1.3 times, but decreasing of hydrogen concentration in 1.45 times, which associated with decreasing of hydrogen saturation limit. Optimal values of temperature $\left(400{ }^{\circ} \mathrm{C}\right)$ and pressure $(2 \mathrm{~atm})$ for pure titanium powder hydrogenation have been determined.
\end{abstract}

\section{Introduction}

One of the important tasks in the hydrogen energy is a task to develop effective hydrogenstorage materials (HSM). The selection of the optimal composition of the HSM and the synthesis parameters is carried out on the basis of data on the interaction of hydrogen with obtained materials. HSM should have high rates of hydrogen adsorption and desorption in the operating conditions, low activation time of adsorption and desorption, cycling stability [1]. Gas-phase hydrogenation is used for evaluation all these properties of synthesized HSM. Hydrogenation parameters such as temperature and pressure play an essential role in the hydrogen interaction with materials [2].

In this work technique for hydrogenation of powder HSM has been developed. Pure titanium powder was changed as a model material as soon as titanium is an important element of many HSM. The influence of hydrogenation parameters on the activation time,

\footnotetext{
Corresponding author: viktor.kudiiarov@gmail.com
} 
the rate of hydrogen sorption and absolute hydrogen concentration in the powder was investigated.

\section{Materials and Methods}

Pure titanium powder was used for investigation and it was put into cuprum capsule with quartz wool for isolation from wall of reaction chamber. Experimental chamber was put into automated complex Gas Reaction Controller LPB by Advanced Materials Research [3]. Then chamber was pumped out and heated with heating rate $6{ }^{\circ} \mathrm{C} / \mathrm{min}$ for needed temperature. The temperature diapason was from 400 to $550{ }^{\circ} \mathrm{C}$ with step $50{ }^{\circ} \mathrm{C}$. Chamber was filled by hydrogen for needed pressure after heating. Pressure diapason was from 0.5 to 2 atm with step $0.5 \mathrm{~atm}$.

Titanium powder was kept in hydrogen medium at constant temperature and pressure until full hydrogenation and then slowly cooled with rate $2{ }^{\circ} \mathrm{C} / \mathrm{min}$ in the hydrogen medium. Hydrogen concentration in powder was measured by volumetric method in the automated complex Gas Reaction Controller and with the help of hydrogen analyzer RHEN602 by LECO.

\section{Results and Discussion}

Hydrogen sorption curves by titanium powder at temperature $400{ }^{\circ} \mathrm{C}$ and pressure $0.5 \mathrm{~atm}$ (a) and at temperature $550{ }^{\circ} \mathrm{C}$ and pressure $2 \mathrm{~atm}(\mathrm{~b})$ are shown on the figure 1.
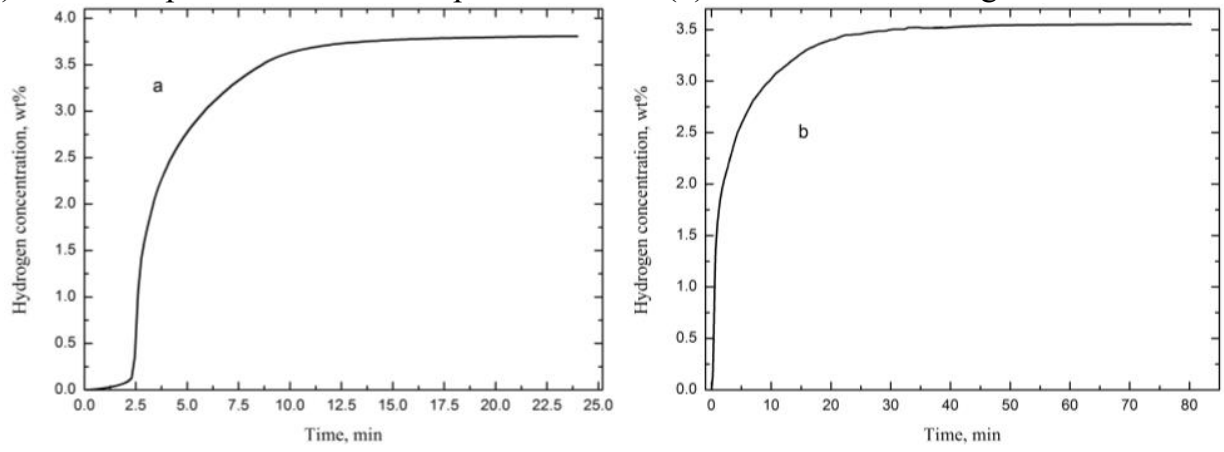

Fig. 1. Hydrogen sorption curves by titanium powder at temperature $400{ }^{\circ} \mathrm{C}$ and pressure $0.5 \mathrm{~atm}$ (a) and at temperature $550^{\circ} \mathrm{C}$ and pressure $2 \mathrm{~atm}(\mathrm{~b})$.

It is seen from figure 1a that at $400{ }^{\circ} \mathrm{C}$ there is a plateau-like section in a sorption curve that is associated with the activation of titanium powder. Than with pressure increasing the time of activation is reduced (figure $2 \mathrm{a}$, the solid line) and at $2 \mathrm{~atm}$ it becomes negligible. Powder activation apparently occurs in the first few seconds of the hydrogen inlet into the chamber at hydrogenation at high temperatures (including $550{ }^{\circ} \mathrm{C}$ ), since there is not the plateau-like section in the sorption curves (figure $1 \mathrm{~b}$ ).

Hydrogen sorption rates have been calculated based on the sorption curves depending on the pressure at $400{ }^{\circ} \mathrm{C}$ (figure $2 \mathrm{a}$, the dotted line) and depending on the temperature at a constant pressure of $2 \mathrm{~atm}$. (figure $2 \mathrm{~b}$ ). 

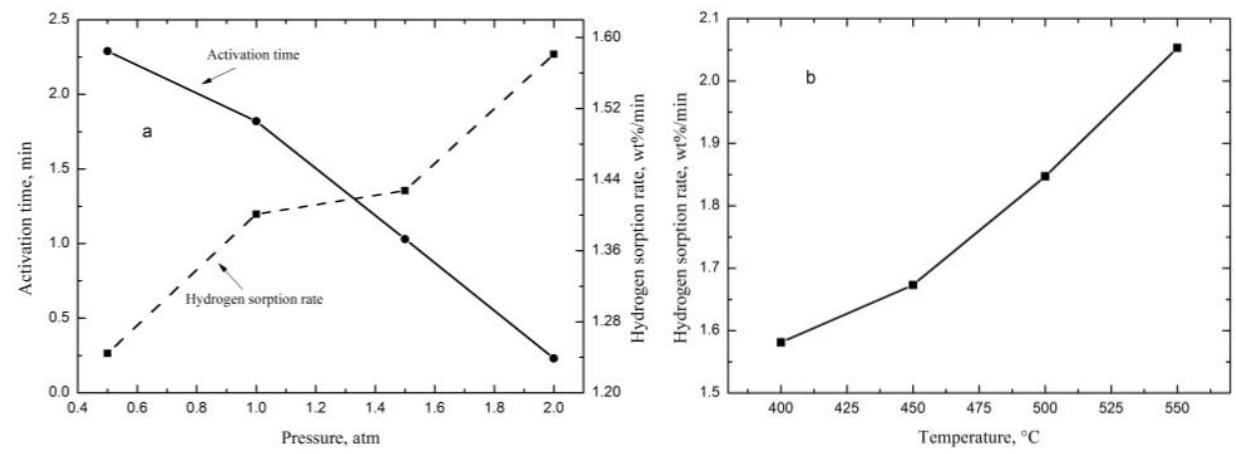

Fig. 2. The dependence of powder activation time (the solid line) and hydrogen sorption rate (the dotted line) on the pressure at constant temperature $400{ }^{\circ} \mathrm{C}$ (a) and dependence of hydrogen sorption rate on temperature at constant pressure $2 \mathrm{~atm}(\mathrm{~b})$.

The opposite effect is observed for the dependence of the sorption rate on pressure and temperature. Pressure and temperature increasing leads to the hydrogen sorption rate increasing. However, if the pressure increases, a slight increase in the absolute hydrogen concentration is observed in the material after the hydrogenation, but the temperature increasing leads to the significant reduction of hydrogen concentration (Table 1), which is associated with a reduction of titanium saturation limit with temperature increasing [4].

Table 1. Hydrogen concentration in titanium powder after hydrogenation at different parameters.

\begin{tabular}{|c|c|c|}
\hline Temperature, ${ }^{\circ} \mathrm{C}$ & Pressure, atm. & Hydrogen concentration, wt.\% \\
\hline 400 & 0.5 & 3.82 \\
\hline 400 & 1 & 3.87 \\
\hline 400 & 1.5 & 3.93 \\
\hline 400 & 2 & 4.01 \\
\hline 450 & 2 & 3.92 \\
\hline 500 & 2 & 3.78 \\
\hline 550 & 2 & 3.51 \\
\hline
\end{tabular}

The optimal parameters of hydrogenation (temperature and pressure) are necessary to determine when working with titanium-based HSM for balance between the rate of hydrogen sorption and absolute concentration. Temperature of $400^{\circ} \mathrm{C}$ and a pressure of 2 atm are optimal for the pure titanium powder.

Preparation of the experimental samples of titanium powder with different hydrogen concentrations in the range of 0.5 to $4 \mathrm{wt} . \%$ have interest for different research. For this reason hydrogenation was performed manually at a constant temperature of $400{ }^{\circ} \mathrm{C}$ and hydrogen manual admitted into the chamber. Experimental samples of pure titanium powder with concentrations ranging from 0.5 to 4 wt. $\%$ were prepared By varying the amount of hydrogen in the reaction chamber.

\section{Conclusion}

The technique for hydrogenation of powder hydrogen-storage materials based on gas-phase hydrogenation method has been developed in this work. The pure titanium powder was changed for investigation as a model material. The using of developed technique allow prepare samples with hydrogen concentration in range from 0.5 to $4 \mathrm{wt} . \%$. In additional, pressure and temperature influence on powder activation time, hydrogen sorption rate and 
hydrogen concentration was investigated. It was showed, that pressure increasing from 0.5 to $2 \mathrm{~atm}$ at constant temperature $400{ }^{\circ} \mathrm{C}$ leads to decreasing of powder activation time from 140 to $15 \mathrm{~s}$. Temperature increasing on 50 and more degrees leads to significant decreasing of powder activation time.

Pressure increasing from 0.5 to 2 atm leads to increasing of hydrogen sorption rate and concentration in 1.27 and 1.19 times respectively. Temperature increasing from 400 to 550 ${ }^{\circ} \mathrm{C}$ leads to increasing of hydrogen sorption rate in 1.3 times, but decreasing of hydrogen concentration in 1.45 times, which associated with decreasing of hydrogen saturation limit.

Thereby, hydrogenation parameters play important role at working with titanium-based hydrogen-storage materials and it optimal values should be determined for balance between hydrogen sorption rate and hydrogen concentration. Temperature $400{ }^{\circ} \mathrm{C}$ and pressure 2 atm are for pure titanium powder such parameters.

\section{Acknowledgements}

This work was funded within the framework of realisation of Strategic Programme on National Research Tomsk Polytechnic University Competitiveness Enhancement in the Group of Top Level World Research and Academic Institutions.

\section{References}

1. B.A. Kolachev, P.E. Shalin, A.A. Ilin, Hydrogen-storage alloys (Metallurgy, Moscow, 1995)

2. L. Schlapbach, A. Züttel, Nat. 414 (2001)

3. V.N. Kudiiarov, A.M. Lider, S.Y. Harchenko, Adv. Mater. Res. 880 (2014)

4. I.P. Chernov, Yu. P. Cherdantsev, Yu. I. Tyurin, Methods for metal-hydrogen systems investigation (Energoatomisdat, Tomsk, 2004) 